\title{
Autophagy in Acute Kidney Injury and Repair
}

\author{
Liyu He $^{\mathrm{a}}$ Man J. Livingston ${ }^{\mathrm{b}}$ Zheng Dong ${ }^{\mathrm{a}} \mathrm{b}$ \\ ${ }^{a}$ Department of Nephrology, The Second Xiangya Hospital, Central South University, Changsha, PR China; \\ ${ }^{b}$ Department of Cellular Biology and Anatomy, Medical College of Georgia, Georgia Regents University and \\ Charlie Norwood VA Medical Center, Augusta, Ga., USA
}

\section{Key Words}

Acute kidney injury · Apoptosis · Autophagy · Fibrosis .

Kidney repair

\section{Abstract}

Acute kidney injury (AKI) is a major kidney disease associated with a poor clinical outcome both in the short and long term. Autophagy is a cellular stress response that plays important roles in the pathogenesis of various diseases. Autophagy is induced in proximal tubules during AKI. A renoprotective role of autophagy in AKI has been demonstrated by pharmacological and genetic inhibition studies. The role of autophagy in kidney recovery and repair from AKI, however, remains largely unknown. A dynamic change in autophagy during the recovery phase of AKI seems to be important for tubular proliferation and repair. In renal fibrosis, autophagy may either promote this via the induction of tubular atrophy and decomposition, or prevent it via effects on the intracellular degradation of excessive collagen. Further research is expected to improve the understanding of the regulation of autophagy in kidney injury and repair, elucidate the patho- logical roles of autophagy in renal fibrosis, and discover therapeutic targets for treating $\mathrm{AKI}$ and preventing its progression to chronic kidney disease.

(c) 2014 S. Karger AG, Basel

\section{Introduction}

Autophagy is an 'autodigestive' process in a cell that promotes the delivery of intracellular components from the cytoplasm to lysosomal or vacuolar compartments for terminal degradation and recycling [1]. In the past decade, there has been an intense interest in elucidating the basic molecular mechanism of autophagy and defining its roles in human health and disease [2]. In renal systems, in vitro cell culture, in vivo animal models, and human kidney studies suggest that autophagy plays an important role in

Targeting Recovery from Acute Kidney Injury: Round Table Conference at the 19th International Conference on Continuous Renal Replacement Therapies (Manchester Grand Hyatt, San Diego, Calif., USA, March 2-3, 2014).

\section{KARGER}

E-Mail karger@karger.com www.karger.com/nec
(C) 2014 S. Karger AG, Basel

$1660-2110 / 14 / 1274-0056 \$ 39.50 / 0$
Zheng Dong, $\mathrm{PhD}$

Department of Cellular Biology and Anatomy Medical College of Georgia, Georgia Regents University 1459 Laney Walker Boulevard, Augusta, GA 30912 (USA)

E-Mail zdong@gru.edu 
the maintenance of renal function under both physiological and pathological conditions [3, 4]. This review summarizes the current understanding of the role and regulation of autophagy in acute kidney injury (AKI) and repair.

\section{Autophagy in AKI}

AKI is a major renal disease that is associated with significant mortality and the development of chronic kidney disease and end-stage renal disease. The pathogenesis of AKI is multifactorial. Pathologically, AKI is characterized by tubular cell injury and death. Recent studies have demonstrated that autophagy is rapidly induced during AKI to protect tubular cells from injury and death [5].

Renal ischemia-reperfusion injury (IRI) is a main cause of AKI that is frequently associated with cardiac surgery, renal vascular obstruction, and kidney transplantation. In 2007, Chien et al. [6] showed that autophagic genes, namely beclin 1 and LC3, were induced during renal IRI in rats. Interestingly, beclin 1 and LC3 expression was shown along with apoptosis in injured renal tubules and $\mathrm{Bcl}-\mathrm{xL}$ could block their expression as well as tubular apoptosis [6]. Subsequent work by Suzuki et al. [7] further showed autophagosome-like structures in renal tubular cells during renal IRI in mice and, notably, also in human kidney transplants. In 2010, we demonstrated autophagy induction during hypoxic incubation of renal tubular cells and renal IRI in mice. The induction was rapid, prior to tissue damage or tubular apoptosis [8]. Consistently, later studies using GFP-LC3 transgenic mice showed an increased formation of GFP-LC3 puncta in proximal tubules during renal IRI $[9,10]$.

Autophagy is also induced in experimental models of nephrotoxic AKI. This has been shown during cisplatin treatment of various renal proximal tubular cell lines [1113]. Autophagy induction was indicated by autophagosome formation and LC3-II accumulation. Similar to hypoxic incubation, autophagy was induced by cisplatin within hours preceding the occurrence of apoptosis. Importantly, detailed examination by electron microscopy revealed a time-dependent increase in autophagic vacuoles in kidney proximal tubular cells after cisplatin treatment of C57BL/6 mice [11]. Cisplatin-induced autophagy was further monitored in kidney tissues of GFP-LC3 mice, consistently unveiling autophagy induction in proximal tubules $[13,14]$.

The role of autophagy in renal IRI was originally suggested to participate in tubular cell death $[6,7]$. For example, Suzuki et al. [7] showed that autophagy inhibitors

Autophagy in Kidney Injury and Repair suppressed $\mathrm{H}_{2} \mathrm{O}_{2}$-induced cell death in $\mathrm{HK} 2$ cells. However, recent studies using pharmacological and particularly genetic approaches have provided compelling evidence for a renoprotective role of autophagy in several experimental models of AKI [5]. We showed that in cultured renal tubular cells, pharmacological inhibitors of autophagy as well as autophagy gene knockdown enhanced apoptosis during hypoxic incubation and anoxiareoxygenation treatment. Importantly, inhibition of autophagy by chloroquine or 3-methyladenine worsened renal IRI in mice, as indicated by renal function, histology, and tubular apoptosis, supporting a protective role of autophagy in ischemic AKI [8]. In a conditional knockout mouse model, Atg5 deficiency in proximal tubules dramatically sensitizes the kidneys to ischemic injury, resulting in impaired kidney function, accumulation of damaged mitochondria as well as increased tubular cell apoptosis and necrosis. These findings further highlight the critical role autophagy plays in maintaining tubular cell integrity and promoting cell survival during ischemic AKI $[9,10]$. A renoprotective role of autophagy has also been shown in cisplatin-induced AKI. Under this condition, inhibition of autophagy by chloroquine aggravated, whereas upregulation of autophagy by rapamycin attenuated renal function loss and tubular damage. Consistently, proximal tubule-specific Atg7 knockout mice exhibited increased renal injury compared to their wild-type littermates [15]. Furthermore, in proximal-tubule specific Atg5 knockout mice, cisplatin induced more severe kidney injury, which was accompanied by a massive accumulation of protein aggregates as well as enhanced DNA damage and p53 activation [14]. In addition, autophagy has been suggested to protect kidneys against septic AKI. In rats subjected to cecal ligation and puncture, autophagy increased transiently in kidneys at the early stage of sepsis and then went down toward to the basal level at the late stage. Interestingly, this decline in the autophagic response is associated with the development of kidney injury induced by sepsis. Moreover, knockdown of Atg7 exaggerated, whereas preincubation of rapamycin diminished tumor necrosis factor- $\alpha$-induced cell death in cultured renal tubular cells [16].

\section{Autophagy in Recovery from AKI}

\section{Dynamic Changes of Autophagy in Renal Tubules} during Injury and Repair

It is noteworthy that in the aforementioned studies induction of autophagy in proximal tubular cells and in 
kidneys is mainly examined at a certain time point of AKI. Using a novel autophagy reporter mouse model in which a tandem RFP-EGFP-LC3 fusion protein is expressed ubiquitously under the CAG promoter, a recent study has revealed the dynamics and flux of autophagy in proximal tubules under stress conditions, including starvation and ischemic AKI [17]. While the EGFP fluorescence is sensitive to the acidic environment in the lysosome, the RFP signal is more stable and remained. Therefore, colocalization of both EGFP and RFP fluorescence indicates early autophagic vesicles such as phagophores and autophagosomes. In contrast, autolysosomes appear red (RFP only) due to quenching of the EGFP fluorescence in the low $\mathrm{pH}$ environment [18]. Under normal conditions, nephrons expressed few EGFP and RFP puncta; however, renal IRI led to an increase in the numbers of both RFP and EGFP puncta 1 day after IRI. The number of EGFP puncta returned to control levels 3 days after IRI, whereas the high levels of RFP puncta persisted till 7 days after reperfusion. These findings suggest that autophagy was initiated 1 day after IRI with increased formation of autophagosomes. As kidneys were recovering from injury on day 3 , autophagosomes proceeded to fuse with lysosomes for clearance, indicating that resolution of autophagy was predominant during the recovery phase. mTOR was activated in proximal tubules after renal IRI and facilitated autophagy resolution. Of note, in cells with inhibited mTOR and persistent RFP puncta, tubular proliferation was inhibited, suggesting that autophagic cells are less likely to divide for tubular repair [17].

\section{Autophagy in Renal Fibrosis}

Although renal tubular cells have the ability to regenerate after injury, tubular repair after severe or episodic AKI is mostly incomplete, resulting in renal interstitial fibrosis $[19,20]$. Induction of autophagy and its contribution to fibrotic diseases has been suggested in the lung, liver, and heart [21-23]. The pathological roles of autophagy in fibrosis in those organs vary greatly depending on the type of cells or tissues and pathological conditions [24]. Evidence thus far regarding autophagy in kidney fibrosis derives mainly from studies using models of unilateral ureteral obstruction and transforming growth factor (TGF) $-\beta[25]$. Under these conditions, involvement of autophagy in either tubular atrophy or degradation of collagen has been suggested, which apparently contributes oppositely to the pathogenesis of renal fibrosis.
Autophagy in Tubular Atrophy during Fibrosis

The role of atrophic changes in renal tubules in peritubular fibrosis has been well documented. Earlier studies in rat models of microembolism- and renal ablation-induced fibrosis showed that focal fibrotic lesions with clusters of atrophic proximal tubules alternated with nonfibrotic areas containing normal tubules [26, 27]. The close spatial relationship between atrophic tubules and the development of interstitial fibrosis was further demonstrated in rat kidneys following IRI [28]. Importantly, proximal tubules in areas that had been previously damaged failed to differentiate. In contrast to surrounding tubules that did recover normally, these atrophic tubules showed significantly decreased expression of differentiation markers such as $\mathrm{Na}^{+}-\mathrm{K}^{+}$-ATPase, ksp-cadherin, and meprin [28]. Myofibroblasts greatly proliferated in the interstitium adjacent to injured and regenerating tubules by 3 days of reperfusion, and, notably, continued to proliferate around the atrophic tubules with undifferentiated tubular epithelium. As a result, by 14 days of reperfusion, foci of tubulointerstitial fibrosis formed around the atrophic tubules [28]. Interestingly, there was a persistent increase in platelet-derived growth factor- $\beta$ chain in atrophic tubules and this phenotype change in tubular cells was associated with an increased expression of platelet-derived growth factor receptor- $\beta$ in adjacent interstitial fibroblasts, suggesting a paracrine ligand-receptor couple that may have been responsible for fibroblast proliferation and extracellular matrix protein deposition in these renal fibrosis models [26-28].

The connection of autophagy and tubular atrophy during renal fibrosis was initially suggested in mice subjected to unilateral ureteral obstruction [29]. In this study, autophagy was activated in obstructed tubules, as indicated by autophagosome accumulation, increased expression of beclin1, and conversion of LC3-I to LC3-II. These changes were accompanied by an increased lysosomal activity, further suggesting induction of autophagic flux in obstructed tubules. Along with autophagy, tubular apoptosis was also induced in obstructed tubules. Importantly, the development of tubular atrophy correlated with autophagy and apoptosis in a time-dependent manner, suggesting that autophagy may act in concert with apoptosis to induce tubular atrophy and nephron loss in this obstructive uropathy [29]. Koesters et al. [30] further suggested the involvement of autophagy in tubular degeneration and fibrosis using a tetracycline-controlled transgenic mouse model that overexpresses TGF- $\beta 1$ in renal tubules. They showed that sustained expression of TGF- $\beta 1$ induced autophagy in tubular cells, as indicated by strong immunostaining of LC3 and formation of autophagic vacuoles un-
He/Livingston/Dong 


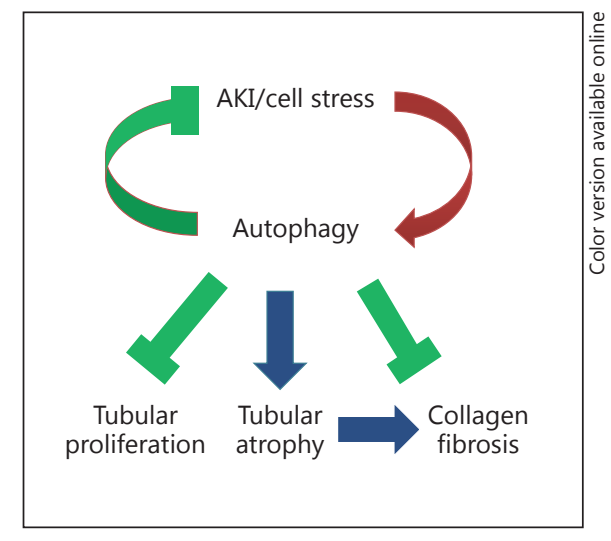

Fig. 1. Diagram depicting the roles of autophagy in AKI and its recovery.

der the electron microscope. Importantly, the tubules with autophagic features were collapsed, with cell debris filling remnant lumina. These tubular changes were associated with widespread peritubular fibrosis. The nuclei of such degenerating cells displayed a normal chromatin pattern without positive TUNEL staining for apoptosis. Together, these results suggest that autophagy may be a main force driving tubular dedifferentiation and decomposition in TGF- $\beta 1$-induced fibrosis [30].

\section{Autophagic Degradation of Collagen I during}

Fibrosis

Intracellular degradation of extracellular matrix proteins, including collagen, by autophagy has been suggested in alveolar epithelial cells and pulmonary fibroblasts of fibrotic lung tissues as well as cardiac fibroblasts in cardiac fibrosis [24]. The negative regulation of matrix proteins by autophagy was recently shown in primary cultured mouse kidney mesangial cells [31]. Both protein and mRNA levels of collagen I were induced by TGF- $\beta 1$ in these cells. Bafilomycin A, an autophagy inhibitor that blocks autolysosomal degradation, further enhanced the protein level of collagen I without affecting mRNA expression. Under this condition, colocalization of collagen I with LC3 and LAMP1 was also increased. Consistent with the pharmacological findings, genetic knockdown of beclin 1 accelerated collagen I protein accumulation by TGF- $\beta 1$. Compared to wild-type cells, mouse kidney mesangial cells obtained from heterozygous beclin1 knockout (beclin1-/-) mice had increased levels of collagen aggregates even under normal conditions, and treatment of TGF- $\beta 1$ further enhanced aggregated collagen I. Togeth- er, these results have revealed a role of autophagy as a protective mechanism to prevent excess collagen accumulation in the kidney [31].

\section{Conclusions}

In conclusion, autophagy is induced in kidneys in response to AKI and protects against kidney injury. During the recovery phase of AKI, resolution of autophagy may promote cell proliferation for tubular regeneration and repair. The role of autophagy in renal fibrosis after AKI is poorly understood. Evidence obtained from recent studies using unilateral ureteral obstruction and TGF- $\beta$ models have demonstrated dual roles of autophagy. On the one hand, persistent activation of autophagy may contribute to tubular atrophy and thereby promote kidney fibrosis. On the other hand, autophagy can prevent fibrosis by mediating intracellular degradation of excessive collagen (fig. 1). Further research should focus on the regulation of autophagy in kidney injury and repair as well as on the role of autophagy in renal fibrosis following AKI. A comprehensive understanding of the regulation and pathological roles of autophagy in AKI and its recovery will facilitate the discovery of genetic and pharmacologic approaches for treating AKI and preventing AKI progression.

\section{Acknowledgments}

This work was supported in part by grants from the National Natural Science Foundation of China (81370791) and the National Institutes of Health and the US Department of Veterans Affairs.

\section{Disclosure Statement}

The authors declared no competing interests.

References 
$>5$ Livingston MJ, Dong Z: Autophagy in acute kidney injury. Semin Nephrol 2014;34:17-26.

6 Chien CT, Shyue SK, Lai MK: Bcl-xL augmentation potentially reduces ischemia/reperfusion induced proximal and distal tubular apoptosis and autophagy. Transplantation 2007;84:1183-1190.

$>7$ Suzuki C, Isaka Y, Takabatake Y, Tanaka H, Koike M, Shibata M, et al: Participation of autophagy in renal ischemia/reperfusion injury. Biochem Biophys Res Commun 2008;368: 100-106.

8 Jiang M, Liu K, Luo J, Dong Z: Autophagy is a renoprotective mechanism during in vitro hypoxia and in vivo ischemia-reperfusion injury. Am J Pathol 2010;176:1181-1192.

9 Kimura T, Takabatake Y, Takahashi A, Kaimori JY, Matsui I, Namba T, et al: Autophagy protects the proximal tubule from degeneration and acute ischemic injury. J Am Soc Nephrol 2011;22:902-913.

$>10$ Liu S, Hartleben B, Kretz O, Wiech T, Igarashi P, Mizushima N, et al: Autophagy plays a critical role in kidney tubule maintenance, aging and ischemia-reperfusion injury. Autophagy 2012;8:826-837.

-11 Periyasamy-Thandavan S, Jiang M, Wei Q, Smith R, Yin XM, Dong Z: Autophagy is cytoprotective during cisplatin injury of renal proximal tubular cells. Kidney Int 2008;74: 631-640.

-12 Yang C, Kaushal V, Shah SV, Kaushal GP: Autophagy is associated with apoptosis in cisplatin injury to renal tubular epithelial cells. Am J Physiol Renal Physiol 2008;294:F777-F787.

$>13$ Inoue K, Kuwana H, Shimamura Y, Ogata K, Taniguchi Y, Kagawa T, et al: Cisplatin-induced macroautophagy occurs prior to apoptosis in proximal tubules in vivo. Clin Exp Nephrol 2010;14:112-122.
14 Takahashi A, Kimura T, Takabatake Y, Namba T, Kaimori J, Kitamura H, et al: Autophagy guards against cisplatin-induced acute kidney injury. Am J Pathol 2012;180:517-525.

15 Jiang M, Wei Q, Dong G, Komatsu M, Su Y, Dong Z: Autophagy in proximal tubules protects against acute kidney injury. Kidney Int 2012;82:1271-1283.

16 Hsiao HW, Tsai KL, Wang LF, Chen YH, Chiang PC, Chuang SM, et al: The decline of autophagy contributes to proximal tubular dysfunction during sepsis. Shock 2012;37:289296.

Li L, Wang ZV, Hill JA, Lin F: New autophagy reporter mice reveal dynamics of proximal tubular autophagy. J Am Soc Nephrol 2014;25: 305-315.

18 Kimura S, Noda T, Yoshimori T: Dissection of the autophagosome maturation process by a novel reporter protein, tandem fluorescenttagged LC3. Autophagy 2007;3:452-460.

19 Venkatachalam MA, Griffin KA, Lan R, Geng H, Saikumar P, Bidani AK: Acute kidney injury: a springboard for progression in chronic kidney disease. Am J Physiol Renal Physiol 2010;298:F1078-F1094.

20 Yang L, Humphreys BD, Bonventre JV: Pathophysiology of acute kidney injury to chronic kidney disease: maladaptive repair. Contrib Nephrol 2011;174:149-155.

21 Hernandez-Gea V, Ghiassi-Nejad Z, Rozenfeld R, Gordon R, Fiel MI, Yue Z, et al: Autophagy releases lipid that promotes fibrogenesis by activated hepatic stellate cells in mice and in human tissues. Gastroenterology 2012;142:938-946.

-22 Yang HZ, Wang JP, Mi S, Liu HZ, Cui B, Yan $\mathrm{HM}$, et al: TLR4 activity is required in the resolution of pulmonary inflammation and fibrosis after acute and chronic lung injury. Am J Pathol 2012;180:275-292.

23 Aránguiz-Urroz P, Canales J, Copaja M, Troncoso R, Vicencio JM, Carrillo C, et al: $\mathrm{Beta}(2)$-adrenergic receptor regulates cardiac fibroblast autophagy and collagen degradation. Biochim Biophys Acta 2011;1812:23-31.
24 Del Principe D, Lista P, Malorni W, Giammarioli AM: Fibroblast autophagy in fibrotic disorders. J Pathol 2013;229:208-220.

25 Ding Y, Choi ME: Regulation of autophagy by TGF-beta: emerging role in kidney fibrosis. Semin Nephrol 2014;34:62-71.

26 Suzuki T, Kimura M, Asano M, Fujigaki Y, Hishida A: Role of atrophic tubules in development of interstitial fibrosis in microembolism-induced renal failure in rat. Am J Pathol 2001;158:75-85.

27 Kimura M, Asano M, Abe K, Miyazaki M, Suzuki T, Hishida A: Role of atrophic changes in proximal tubular cells in the peritubular deposition of type IV collagen in a rat renal ablation model. Nephrol Dial Transplant 2005;20: 1559-1565.

28 Geng H, Lan R, Wang G, Siddiqi AR, Naski $\mathrm{MC}$, Brooks AI, et al: Inhibition of autoregulated TGFbeta signaling simultaneously enhances proliferation and differentiation of kidney epithelium and promotes repair following renal ischemia. Am J Pathol 2009;174: 1291-1308.

29 Li L, Zepeda-Orozco D, Black R, Lin F: Autophagy is a component of epithelial cell fate in obstructive uropathy. Am J Pathol 2010; 176:1767-1778.

30 Koesters R, Kaissling B, Lehir M, Picard N, Theilig F, Gebhardt R, et al: Tubular overexpression of transforming growth factor-betal induces autophagy and fibrosis but not mesenchymal transition of renal epithelial cells. Am J Pathol 2010;177:632-643.

-31 Kim SI, Na HJ, Ding Y, Wang Z, Lee SJ, Choi ME: Autophagy promotes intracellular degradation of type I collagen induced by transforming growth factor (TGF)-beta1. J Biol Chem 2012;287:11677-11688. 